\title{
Hypoparathyroidism, CTCAE
}

National Cancer Institute

\section{Source}

National Cancer Institute. Hypoparathyroidism, CT CAE. NCI Thesaurus. Code C143572.

A disorder characterized by a decrease in production of parathyroid hormone by the parathyroid glands. 